\title{
A stochastic analysis for a triple delayed SIQR epidemic model with vaccination and elimination strategies
}

\author{
Mohamed El Fatini ${ }^{1}$ (D) $\cdot$ Roger Pettersson $^{2} \cdot$ Idriss Sekkak $^{1} \cdot$ Regragui Taki $^{3}$
}

Received: 21 January 2020 / Revised: 09 May 2020 / Accepted: 08 June 2020 / Published online: 26 June 2020

c) Korean Society for Informatics and Computational Applied Mathematics 2020

\begin{abstract}
In this paper, a delayed SIQR epidemic model with vaccination and elimination hybrid strategies is analysed under a white noise perturbation. We prove the existence and the uniqueness of a positive solution. Afterwards, we establish a stochastic threshold $\mathcal{R}_{S}$ in order to study the extinction and persistence in mean of the stochastic epidemic system. Then we investigate the existence of a stationary distribution for the delayed stochastic model. Finally, some numerical simulations are presented to support our theoretical results.
\end{abstract}

Keywords Extinction · Persistence in mean · Delay - White noise - Epidemic model · Stationary distribution

Mathematics Subject Classification 92B05 · 60G51 · 60H30 · 60G57

\section{Introduction}

Governments has always made the public health policy as a priority and adopted decisions, plans and actions to save human lives from deadly infectious diseases. For

Mohamed El Fatini

melfatini@gmail.com

Roger Pettersson

roger.pettersson@1nu.se

Idriss Sekkak

idrisssekkak@gmail.com

Regragui Taki

taki.reg@gmail.com

1 Département de Mathématiques, Faculté des Sciences, Université Ibn Tofail, BP 133, Kénitra, Morocco

2 Department of Mathematics, Linnaeus University, 35195 Växjö, Sweden

3 Chouaib Doukkali University EST Sidi Bennour, El Jadida, Morocco 
this matter, computational biologists study the dynamics of epidemics to prevent and control the infection from spreading in the population $[1,2,6]$. Historically, in the 14 th century, the authorities of the city of Venice have installed a measure of isolation to enter and exit its ports, where every crew in every ship was inspected, once the whole personal have no symptoms, then they could be cleared out to land. This idea is adopted as a major measure to prevent infectious diseases such as Ebola and Malaria from spreading. Nowadays, every well equipped hospital has a number of rooms and halls dedicated for the isolation of infected individuals. In addition, the authorities can put their people under quarantine. This decision has a significant impact on the basic reproduction number, which leads us to the extinction of the infectious disease. Recently, the quarantine measure has proven to be efficient in the extinction of the COVID19 disease in China, which let many countries to adopt this strategy in absence of vaccine or a cure to the new Corona virus. In order to understand the effect of quarantine on the behavior of the epidemics, Heathcote [13] proposed a model with quarantine to describe isolated individuals in the compartmental model followed by other papers such as [5,27].

On the other hand, a new type of delayed stochastic models are proposed to describe the role of time delay in reality which leads to a more complex behavior of the stability of the dynamic system. This concept is described as a temporary immunity in $[1,10,12]$ and as a vaccine effect in [8,9]. However, the temporary immunity can impact also the quarantined individuals. Therefore delayed stochastic epidemic models are concerning the persistence in mean, ultimate boundedness and permanence [5] or the asymptotic behavior around the equilibrium points of the infectious diseases models. In [15], the authors obtained sufficient conditions for the existence and uniqueness of stationary distribution for a delayed stochastic differential equations with positivity constraints and applied theoretical results for biochemical reaction system. Also, Zhang and Yuan [28] used Lyapunov analysis method to investigate the existence of stationary distribution of a stochastic delayed chemostat model. Therefore, it is important to investigate a relatively weak characteristic for a delayed stochastic epidemic model.

In recent years, epidemic models with quarantine for the have been proposed [23, $25,26]$. On the other hand, for the modelling of the environemental noise. In $[3,4,16,20$, 24,29] perturbed systems with white noise and isolation for population dynamics are considered. Liu et al. [22] investigated a SIQR epidemic model with telegraph noise to study the influence of isolation for a compartmental SIR model, where they established a stochastic threshold for the extinction, persistence in mean and obtained sufficient conditions for the existence of positive recurrence of the solutions. By constructing a suitable stochastic Lyapunov function to the epidemic model with regime switching.

Therefore, in order to reflect more the reality we introduced the notion of delay, vaccine and elimination in an SIQR epidemic model. Hence, we propose the following triple delayed SIQR epidemic model with vaccination and isolation strategies

$$
\begin{aligned}
\dot{S}= & A-\beta S(t) I(t)-(\mu+p) S(t)+p S\left(t-\tau_{1}\right) e^{-\mu \tau_{1}} \\
& +\gamma I\left(t-\tau_{2}\right) e^{-\mu \tau_{2}}+\varepsilon Q\left(t-\tau_{3}\right) e^{-\mu \tau_{3}} \\
\dot{I}= & \beta S(t) I(t)-\left(\mu+\alpha_{1}+\delta+\gamma\right) I(t) \\
\dot{Q}= & \delta I(t)-\left(\mu+\alpha_{2}+\varepsilon\right) Q(t)
\end{aligned}
$$




$$
\begin{aligned}
\dot{R}= & \gamma I(t)+p S(t)+\varepsilon Q(t)-\mu R(t)-p S\left(t-\tau_{1}\right) e^{-\mu \tau_{1}} \\
& -\gamma I\left(t-\tau_{2}\right) e^{-\mu \tau_{2}}-\varepsilon Q\left(t-\tau_{3}\right) e^{-\mu \tau_{3}}
\end{aligned}
$$

The compartments susceptible, infected, quarantined or isolated, and recovered are denoted by $S(t), I(t), Q(t)$ and $R(t)$, respectively. The parameter $A$ represents the population recruitment rate, $\mu$ denotes the natural death rate of $S, I, Q$ and $R$ compartments, $\beta$ denotes the transmission coefficient from susceptible to infected individuals, $\gamma$ describes the recovery rate of the infective individuals, $\alpha_{1}$ and $\alpha_{2}$ represents the death rate for infected and quarantined individuals because of infection complications, $p$ stands for the proportional coefficient of vaccinated for the susceptible, $\delta$ denotes the rate of infectious individuals who were isolated, $\varepsilon$ represents the recovered people coming from isolation. The time $\tau_{1}>0$ represents the delay for the efficiency of vaccine. The term $S\left(t-\tau_{1}\right) e^{-\mu \tau_{1}}$ reflects the fact that some individuals remains susceptible even after the vaccine for a specific time. The time $\tau_{2}>0$ is the length of the immunity period. The term $I\left(t-\tau_{2}\right) e^{-\mu \tau_{2}}$ represents the individuals who became susceptible because of the lose of immunity for a specific time. The time $\tau_{3}>0$ denotes the delay for isolated individuals to get back their immunity. The term $Q\left(t-\tau_{3}\right) e^{-\mu \tau_{3}}$ represents the individuals coming out from isolation with immunity impairment. The basic reproduction number of the system (1) is

$$
\mathcal{R}_{0}=\frac{\beta A}{\left(\mu+p\left(1-e^{-\mu \tau_{1}}\right)\right)\left(\mu+\alpha_{1}+\delta+\gamma\right)} .
$$

Next, we establish the following delayed stochastic SIQR epidemic model with vaccination and elimination strategies

$$
\begin{aligned}
d S= & {\left[A-\beta S(t) I(t)-(\mu+p) S(t)+p S\left(t-\tau_{1}\right) e^{-\mu \tau_{1}}+\gamma I\left(t-\tau_{2}\right) e^{-\mu \tau_{2}}\right.} \\
& \left.+\varepsilon Q\left(t-\tau_{3}\right) e^{-\mu \tau_{3}}\right] d t,+\sigma_{1} S(t) d B_{1}(t), \\
d I= & {\left[\beta S(t) I(t)-\left(\mu+\alpha_{1}+\delta+\gamma\right) I(t)\right] d t+\sigma_{2} I(t) d B_{2}(t), } \\
d Q= & {\left[\delta I(t)-\left(\mu+\alpha_{2}+\varepsilon\right) Q(t)\right] d t+\sigma_{3} Q(t) d B_{3}(t), } \\
d R= & {\left[\gamma I(t)+p S(t)+\varepsilon Q(t)-\mu R(t)-p S\left(t-\tau_{1}\right) e^{-\mu \tau_{1}}\right.} \\
& \left.-\gamma I\left(t-\tau_{2}\right) e^{-\mu \tau_{2}}-\varepsilon Q\left(t-\tau_{3}\right) e^{-\mu \tau_{3}}\right] d t+\sigma_{4} R(t) d B_{4}(t),
\end{aligned}
$$

where, $B_{i}(t)$ are independent standard Brownian motions defined on a complete probability space $(\Omega, \mathcal{F}, \mathbb{P})$ with a filtration $\left\{\mathcal{F}_{t}\right\}_{t \geq 0}$ satisfying the usual conditions and $\sigma_{i}$ for $i=1,2,3,4$ represent the volatility perturbations.

We define the differential operator $L$, associated with the following general $d$ dimensional stochastic system

$$
\mathrm{d} X(t)=F(t, X(t), X(t-\tau) \mathrm{d} t+G(t, X(t)) \mathrm{d} B(t), \quad \text { for all } t \geq-\tau, \tau \geq 0
$$

with the initial condition $X(s)=\eta(s)$ for $s \in[-\tau, 0], \eta \in C\left([-\tau, 0] ; \mathbb{R}_{+}^{d}\right)$ and $\eta(s)>0$, where $F(t, X(t), X(t-\tau))$ is a function on $\mathbb{R}^{d}$ defined in $\left[-\tau,+\infty\left[\times \mathbb{R}^{d}\right.\right.$, $G(t, X(t))$ is a $d \times m$ matrix, $F$ and $G$ are locally Lipschitz functions in $x$ and $B(t)$ 
is an $d$-dimensional Wiener process. The differential operator $L$, acts on a function $V \in C^{1,2}\left(C\left([-\tau, 0] ; \mathbb{R}_{+}^{d}\right) \times[-\tau, \infty) ; \mathbb{R}_{+}\right)$, as follows

$$
\begin{aligned}
L V(t, X)= & V_{t}(t, X)+V_{X}(t, X) F(t, X(t), X(t-\tau) \\
& +\frac{1}{2} \operatorname{trace}\left[G^{T}(t, X) V_{X X}(X, t) G(X, t)\right] .
\end{aligned}
$$

By Itô's formula

$$
d V(t, x(t))=L V(t, X(t)) \mathrm{d} t+V_{X}(t, X(t)) G(t, X(t)) \mathrm{d} B(t),
$$

where

$$
V_{t}=\frac{\partial V}{\partial t}, \quad V_{x}=\left(\frac{\partial V}{\partial x_{1}}, \frac{\partial V}{\partial x_{2}}, \ldots, \frac{\partial V}{\partial x_{d}}\right) \quad V_{x x}=\left(\frac{\partial V^{2}}{\partial x_{i} \partial x_{j}}\right)_{d \times d}
$$

For any $X \in \mathbb{R}^{3}$, the norm $|X|$, as usual, is given by $|X|=\sqrt{X_{1}^{2}+X_{2}^{2}+X_{3}^{2}}$.

This work is organized as follows. In Sect. 2, Existence and uniqueness of a global positive solution is shown. In Sect. 3, the stochastic threshold is investigated between the extinction and persistence in man. In Sect. 4, we prove sufficient conditions for the existence of a unique stationary distribution for the delayed stochastic SIQR epidemic model. In Sect. 5, numerical simulations are given to support the theoretical results.

\section{Existence and uniqueness of the global positive solution}

In this section, we prove that the model (3) has a local positive solution. Then we investigate the global positivity of the solution.

Throughout this work, we will reduce our stochastic system (3) using the three first equations since they do not depend on $R(t)$. The fourth equation can be dropped without loss of generality.

Let $\tau=\max \left\{\tau_{1}, \tau_{2}, \tau_{3}\right\}$. We denote

$$
\mathbb{R}_{+}^{3}=\left\{(S, I, Q) \in \mathbb{R}^{3}: S>0, I>0, Q>0\right\}
$$

and let $C=C\left([-\tau, 0], \mathbb{R}_{+}^{3}\right)$ be the Banach space of continuous functions mapping from the interval $[-\tau, 0]$ into $\mathbb{R}_{+}^{3}$ equipped by the norm $\|\phi\|=\sup _{-\tau \leq \theta \leq 0}|\phi(\theta)|$. We set the initial conditions of system (3) to be

$$
\begin{aligned}
& S(\theta)=\phi_{1}(\theta), \quad I(\theta)=\phi_{2}(\theta), \quad Q(\theta)=\phi_{3}(\theta), \\
& \phi_{i}(\theta)>0, \quad \theta \in[-\tau, 0], \quad i=1,2,3, \\
& \left(\phi_{1}, \phi_{2}, \phi_{3}\right) \in C .
\end{aligned}
$$


Theorem 1 For any given initial value $(S(0), I(0), Q(0)) \in \mathbb{R}_{+}^{3}$, there is a positive unique solution $(S(t), I(t), Q(t))$ of model (3) on $t \geq 0$ and the solution will remain in $\mathbb{R}_{+}^{3}$ with probability 1 .

Proof Since the coefficients of the system (3) are locally Lipschitz continuous, for any given initial value $(S(0), I(0), Q(0)) \in \mathbb{R}_{+}^{3}$ there is a unique local solution positive $(S(t), I(t), Q(t))$ on $t \in\left[\tau, \tau_{e}\right)$, where $\tau=\max \left\{\tau_{1}, \tau_{2}, \tau_{3}\right\}$ and $\tau_{e}$ is the explosion time. Now, we show that the solution is global. We have only to prove that $\tau_{e}=\infty$ a.s. Consider an $\varepsilon_{0}>0$ such that $S(0)>\varepsilon_{0}, I(0)>\varepsilon_{0}, Q(0)>\varepsilon_{0}$, then we define the stopping time as follows

$$
\tau_{\tilde{\varepsilon}}=\inf \left\{t \in\left[0, \tau_{e}\right): S(t) \leq \tilde{\varepsilon} \text {, or } I(t) \leq \tilde{\varepsilon} \text {, or } Q(t) \leq \tilde{\varepsilon}\right\}, \quad \forall \tilde{\varepsilon}>0 \text { such that } \tilde{\varepsilon} \leq \varepsilon_{0} \text {. }
$$

Throughout this paper we set $\inf \emptyset=\infty$ ( $\emptyset$ denotes the empty set). It's clear that, $\tau_{\tilde{\varepsilon}}$ is increasing as $\tilde{\varepsilon} \rightarrow 0$. Set $\tau_{0}=\lim _{\tilde{\varepsilon} \rightarrow 0} \tau_{\tilde{\varepsilon}}$. Obviously $\tau_{0} \leq \tau_{e}$ a.s. If $\tau_{0}=\infty$ a.s. is true, then $\tau_{e}=\infty$ a.s. and $(S(t), I(t), Q(t)) \in \mathbb{R}_{+}^{3}$ a.s. for $t \geq 0$. In other words, to complete the proof it is required to show that $\tau_{0}=\infty$ a.s. If this statement is false, then there exist a pair of constants $T>0$ and $\delta \in(0,1)$ such that $\mathcal{P}\left\{\tau_{0} \leq T\right\}>\delta$. Thus there is an $\varepsilon_{1}>0$ such that

$$
\mathcal{P}\left\{\tau_{\tilde{\varepsilon}} \leq T\right\} \geq \delta \quad \forall \tilde{\varepsilon} \leq \varepsilon_{1}
$$

Consider the $C^{2}$-function $V_{1}: \mathbb{R}_{+}^{3} \rightarrow \mathbb{R}$ as follows

$V_{1}(S, I, Q)=\log S(t)+\log I(t)+\log Q(t)-\log \phi_{1}(\theta)-\log \phi_{2}(\theta)-\log \phi_{3}(\theta)$,

Applying Itô's formula on (6) for all $t \in\left[0, \tau_{\tilde{\varepsilon}}\right.$ ) and all $\omega \in\left\{\tau_{\tilde{\varepsilon}}<T\right\}$, we obtain

$$
\begin{aligned}
& V_{1}(S, I, Q) \\
&=\int_{0}^{t}\left[\frac{A}{S(t)}-(\mu+p)-\beta I(t)+\frac{p S\left(s-\tau_{1}\right) e^{-\mu \tau_{1}}}{S(s)}+\frac{\gamma I\left(s-\tau_{2}\right) e^{-\mu \tau_{2}}}{S(s)}\right. \\
&\left.\quad+\frac{\varepsilon Q\left(s-\tau_{3}\right) e^{-\mu \tau_{3}}}{S(s)}-\frac{\sigma_{1}^{2}}{2}\right] d s+\int_{0}^{t}\left[\beta S(t)-\left(\mu+\alpha_{1}+\delta+\gamma\right)-\frac{\sigma_{2}^{2}}{2}\right] d s \\
& \quad+\int_{0}^{t}\left[\frac{\delta I(s)}{Q(s)}-\left(\mu+\alpha_{2}+\varepsilon\right)-\frac{\sigma_{3}^{2}}{2}\right] d s+\sigma_{1} B_{1}(t)+\sigma_{2} B_{2}(t)+\sigma_{3} B_{3}(t) \\
& \geq-\int_{0}^{t}\left[3 \mu+p+\alpha_{1}+\alpha_{2}+\delta+\gamma+\varepsilon+\frac{\sigma_{1}^{2}+\sigma_{2}^{2}+\sigma_{3}^{2}}{2}+\beta I(s)\right] d s+\sigma_{1} B_{1}(t) \\
&+\sigma_{2} B_{2}(t)+\sigma_{3} B_{3}(t) .
\end{aligned}
$$

According to the stopping time $\tau_{\tilde{\varepsilon}}$, for almost all $\omega$ in $\left\{\tau_{\tilde{\varepsilon}}<T\right\}$ at least one component of $\left(S\left(\tau_{\tilde{\varepsilon}}\right), I\left(\tau_{\tilde{\varepsilon}}\right), Q\left(\tau_{\tilde{\varepsilon}}\right)\right)$ is equal to $\tilde{\varepsilon}$. Thus,

$$
\lim _{\tilde{\varepsilon} \rightarrow 0} V_{1}\left(S\left(\tau_{\tilde{\varepsilon}}\right), I\left(\tau_{\tilde{\varepsilon}}\right), Q\left(\tau_{\tilde{\varepsilon}}\right)\right)=-\infty
$$


Letting $t \rightarrow \tau_{\tilde{\varepsilon}}$ in (7), we obtain

$$
\begin{gathered}
-\left(3 \mu+p+\alpha_{1}+\alpha_{2}+\delta+\gamma+\varepsilon+\frac{1}{2}\left(\sigma_{1}^{2}+\sigma_{2}^{2}+\sigma_{3}^{2}\right)\right) \tau_{\tilde{\varepsilon}} \\
-\beta \int_{0}^{\tau_{\tilde{\varepsilon}}} I(s) d s+\sigma_{1} B_{1}\left(\tau_{\tilde{\varepsilon}}\right)+\sigma_{2} B_{2}\left(\tau_{\tilde{\varepsilon}}\right)+\sigma_{3} B_{3}\left(\tau_{\tilde{\varepsilon}}\right)>-\infty .
\end{gathered}
$$

From (8) and extending $\tilde{\varepsilon}$ to zero in (9) contradict our assumption, consequently, $\tau_{0}=\tau_{e}=+\infty$ a.s.

\section{Investigation of a stochastic threshold}

In this section, we investigate a stochastic threshold for the extinction and the persistence in mean which represent an important issues to study the dynamics of the disease. Firstly, we will focus on the following lemmas before investigating a stochastic threshold of the stochastic system (3).

Lemma 1 Let $(S(t), I(t), Q(t))$ be a solution of system (3) with any initial value $\left(S\left(\xi_{1}\right) \geq 0, I\left(\xi_{2}\right) \geq 0, Q\left(\xi_{3}\right)\right) \geq 0$ for all $\xi_{1} \in\left[-\tau_{1}, 0\right), \xi_{2} \in\left[-\tau_{2}, 0\right), \xi_{3} \in\left[-\tau_{3}, 0\right)$ with $S(0)>0, I(0)>0, Q(0)>0$, then

$$
\begin{aligned}
& \lim _{t \rightarrow \infty} \frac{S(t)+I(t)+Q(t)+p S\left(t-\tau_{1}\right) e^{-\mu \tau_{1}}+\gamma I\left(t-\tau_{2}\right) e^{-\mu \tau_{2}}+\varepsilon Q\left(t-\tau_{3}\right) e^{-\mu \tau_{3}}}{t} \\
& \quad=0 \text { a.s }
\end{aligned}
$$

Moreover

$$
\begin{aligned}
& \lim _{t \rightarrow \infty} \frac{S(t)}{t}=0, \quad \lim _{t \rightarrow \infty} \frac{I(t)}{t}=0, \quad \lim _{t \rightarrow \infty} \frac{Q(t)}{t}=0, \quad \lim _{t \rightarrow \infty} \frac{e^{-\mu t} \int_{t-\tau_{1}}^{t} e^{\mu s} S(s) d s}{t}=0 \\
& \lim _{t \rightarrow \infty} \frac{e^{-\mu t} \int_{t-\tau_{2}}^{t} e^{\mu s} I(s) d s}{t}=0, \quad \lim _{t \rightarrow \infty} \frac{e^{-\mu t} \int_{t-\tau_{3}}^{t} e^{\mu s} Q(s) d s}{t}=0 \text { a.s. }
\end{aligned}
$$

Proof Let

$$
\begin{aligned}
V_{1}(t)= & S(t)+I(t)+Q(t)+p e^{-\mu t} \int_{t-\tau_{1}}^{t} e^{\mu s} S(s) d s+\gamma e^{-\mu t} \int_{t-\tau_{2}}^{t} e^{\mu s} I(s) d s \\
& +\varepsilon e^{-\mu t} \int_{t-\tau_{3}}^{t} e^{\mu s} Q(s) d s .
\end{aligned}
$$

Define

$$
V_{2}\left(V_{1}\right)=\left(1+V_{1}\right)^{\theta},
$$


where $\theta$ is a positive constant to be determined later. Applying Itô's formula on $V_{2}$, we get

$$
\begin{aligned}
d V_{2}\left(V_{1}\right)= & \mathcal{L} V_{2}\left(V_{1}\right) d t+\theta\left(1+V_{1}\right)^{\theta-1}\left(\sigma_{1} S(t) d B_{1}(t)+\sigma_{2} I(t) d B_{2}(t)\right. \\
& \left.+\sigma_{3} Q(t) d B_{3}(t)\right)
\end{aligned}
$$

where

$$
\begin{aligned}
& \mathcal{L} V_{2}\left(V_{1}\right) \\
&= \theta\left(1+V_{1}\right)^{\theta-1}\left[A-\mu S(t)-\left(\mu+\alpha_{1}\right) I(t)-\left(\mu+\alpha_{2}\right) Q(t)\right. \\
&\left.-\mu p e^{-\mu t} \int_{t-\tau_{1}}^{t} e^{\mu s} S(s) d s-\mu \gamma e^{-\mu t} \int_{t-\tau_{2}}^{t} e^{\mu s} I(s) d s-\mu \varepsilon e^{-\mu t} \int_{t-\tau_{3}}^{t} e^{\mu s} Q(s) d s\right] \\
&+\frac{\theta(\theta-1)}{2}\left(1+V_{1}\right)^{\theta-2}\left(\sigma_{1}^{2} S^{2}+\sigma_{2}^{2} I^{2}+\sigma_{3}^{2} Q^{2}\right) \\
& \leq \theta\left(1+V_{1}\right)^{\theta-2}\left\{\left(1+V_{1}\right)\left[A-\mu V_{1}-\alpha_{1} I(t)-\alpha_{2} Q(t)\right]\right. \\
&\left.+\frac{\theta-1}{2}\left(\sigma_{1}^{2} S^{2}+\sigma_{2}^{2} I^{2}+\sigma_{3}^{2} Q^{2}\right)\right\} .
\end{aligned}
$$

Let $\sigma^{2}=\sigma_{1}^{2} \vee \sigma_{2}^{2} \vee \sigma_{3}^{2}$. Therefore, we obtain

$$
\begin{aligned}
\mathcal{L} V_{2}\left(V_{1}\right) & \leq \theta\left(1+V_{1}\right)^{\theta-2}\left\{\left(1+V_{1}\right)\left[A-\mu V_{1}\right]+\left(\frac{\theta-1}{2}\right) \sigma^{2} V_{1}^{2}\right\} \\
& \leq \theta\left(1+V_{1}\right)^{\theta-2}\left\{-\left(\mu-\sigma^{2} \frac{\theta-1}{2}\right) V_{1}^{2}+(A-\mu) V_{1}+A\right\} .
\end{aligned}
$$

Choose $\theta>1$ such that $\mu-\left(\frac{\theta-1}{2}\right) \sigma^{2}:=v>0$ then

$$
\mathcal{L}\left(V_{2}\left(V_{1}\right)\right) \leq \theta\left(1+V_{1}\right)^{\theta-2}\left\{-v V_{1}^{2}+(A-\mu) V_{1}+A\right\} .
$$

Yields that

$$
\begin{aligned}
d\left(V_{2}\left(V_{1}\right)\right) \leq & \theta\left(1+V_{1}\right)^{\theta-2}\left[-v V_{1}^{2}+(A-\mu) V_{1}+A\right] d t \\
& +\theta\left(1+V_{1}\right)^{\theta-1}\left[\sigma_{1} S d B_{1}(t)+\sigma_{2} I d B_{2}(t)+\sigma_{3} Q d B_{3}(t)\right]
\end{aligned}
$$

Therefore, for $0 \leq k \leq v \theta$, we get

$$
E\left(e^{k t} V_{2}\left(V_{1}\right)\right)=V_{2}\left(V_{1}(0)\right)+E \int_{0}^{t} \mathcal{L}\left(e^{k s} V_{2}\left(V_{1}(s)\right)\right) d s,
$$

where

$$
\begin{aligned}
\mathcal{L}\left(e^{k t} V_{2}\left(V_{1}\right)\right) & =k e^{k t} V_{2}\left(V_{1}\right)+e^{k t} \mathcal{L} V_{2}\left(V_{1}\right) \\
& \leq \theta e^{k t}\left(1+V_{1}\right)^{\theta-2}\left\{\frac{k}{\theta}\left(1+V_{1}\right)^{2}-v V_{1}^{2}+(A-\mu) V_{1}+A\right\}
\end{aligned}
$$




$$
\begin{aligned}
& =\theta e^{k t}\left(1+V_{1}\right)^{\theta-2}\left(-\left(v-\frac{k}{\theta}\right) V_{1}^{2}+\left(A-\mu+\frac{2 k}{\theta}\right) V_{1}+A+\frac{k}{\theta}\right) \\
& \leq \theta e^{k t} H,
\end{aligned}
$$

with

$$
H:=\sup _{V_{1} \in \mathbb{R}_{+}}\left(1+V_{1}\right)^{\theta-2}\left[-\left(v-\frac{k}{\theta}\right) V_{1}^{2}+\left(A-\mu+\frac{2 k}{\theta}\right) V_{1}+A+\frac{k}{\theta}\right] .
$$

Accordingly to (14), we get

$$
E\left[e^{k t}\left(1+V_{1}(t)\right)^{\theta}\right] \leq\left(1+V_{1}(0)\right)^{\theta}+\frac{\theta H}{k} e^{k t}
$$

Knowing that $V_{1}(t)$ is continuous, yields that there is a positive constant $K$ such that

$$
E\left[\left(1+V_{1}(t)\right)^{\theta}\right] \leq K \text { for all } t \geq 0
$$

Using (13) and according to Burkholder-Davis-Gundy inequality, for a sufficiently small $\varrho>0, \quad k=1,2, \ldots$ and for a positive constant $c_{1}$, we have

$$
E\left[\sup _{k \varrho \leq t \leq(k+1) \varrho}\left(1+V_{1}(t)\right)^{\theta}\right] \leq E\left[\left(1+V_{1}(k \varrho)\right)^{\theta}+\Upsilon_{1}+\Upsilon_{2}\right.
$$

in which

$$
\begin{aligned}
\Upsilon_{1} & =E\left[\sup _{k \varrho \leq t \leq(k+1) \varrho}\left|\int_{k \varrho}^{t} \theta\left(1+V_{1}(s)\right)^{\theta-2}\left[-v V_{1}(s)^{2}+(A-\mu) V_{1}(s)+A\right] d s\right|\right] \\
& \leq c_{1} E\left[\int_{k \varrho}^{(k+1) \varrho}\left(1+V_{1}(s)\right)^{\theta} d s\right] \\
& \leq c_{1} \varrho E\left[\sup _{k \varrho \leq t \leq(k+1) \varrho}\left(1+V_{1}(t)\right)^{\theta}\right],
\end{aligned}
$$

and

$$
\begin{aligned}
\Upsilon_{2} & =E\left[\sup _{k \varrho \leq t \leq(k+1) \varrho} \mid \int_{k \varrho}^{t} \theta\left(1+V_{1}(s)\right)^{\theta-1}\left(\sigma_{1} S(s) d B_{1}(s)+\sigma_{2} I(s) d B_{2}(s)+\sigma_{3} Q(s) d B_{3}(s) \mid\right]\right. \\
& \leq \sqrt{32} E\left[\int_{k \varrho}^{(k+1) \varrho} \theta^{2}\left(1+V_{1}(s)\right)^{2(\theta-1)}\left(\sigma_{1}^{2} S^{2}(s)+\sigma_{2}^{2} I^{2}(s)+\sigma_{3} Q^{2}(s)\right) d s\right]^{\frac{1}{2}} \\
& \leq \sqrt{32} \theta \sigma \varrho^{\frac{1}{2}} E\left[\sup _{k \varrho \leq t \leq(k+1) \varrho}\left(1+V_{1}(t)\right)^{\theta}\right] .
\end{aligned}
$$


Thus

$$
\begin{aligned}
& E\left[\sup _{k \varrho \leq t \leq(k+1) \varrho}\left(1+V_{1}(t)\right)^{\theta}\right] \\
& \leq E\left[\left(1+V_{1}(k \varrho)\right)^{\theta}\right]+\left[c_{1} \varrho+\sqrt{32} \theta \sigma \delta^{\frac{1}{2}}\right] E\left[\sup _{k \varrho \leq t \leq(k+1) \varrho}\left(1+V_{1}(t)\right)^{\theta}\right] .
\end{aligned}
$$

We choose $\varrho>0$ as $c_{1} \varrho+\sqrt{32} \theta \sigma \varrho^{\frac{1}{2}} \leq \frac{1}{2}$ and from (16) we have

$$
E\left[\sup _{k \varrho \leq t \leq(k+1) \varrho}\left(1+V_{1}\right)^{\theta}\right] \leq 2 K
$$

Choosing $\varepsilon_{u}>0$ as an arbitrary number and applying Chebyshev's inequality, we get

$$
\begin{aligned}
P\left\{\sup _{k \varrho \leq t \leq(k+1) \varrho}\left(1+V_{1}\right)^{\theta}>(k \varrho)^{1+\varepsilon_{u}}\right\} & \leq \frac{E\left[\sup _{k \varrho \leq t \leq(k+1) \varrho}\left(1+V_{1}(t)\right)^{\theta}\right]}{(k \varrho)^{1+\varepsilon_{u}}} \\
& \leq \frac{2 K}{(k \varrho)^{1+\varepsilon_{u}}} .
\end{aligned}
$$

For almost all $\sigma \in \Omega$ and by Borel-Cantelli's lemma, we have

$$
\sup _{k \varrho \leq t \leq(k+1) \varrho}\left(1+V_{1}(t)\right)^{\theta} \leq(k \varrho)^{1+\varepsilon_{u}}
$$

holds for all finite $k$. Therefore, there exists for almost all $\omega \in \Omega$, a random integer $k_{0}(\omega)$, where (17) is satisfied for $k \geq k_{0}$. Thus, for almost all $\omega \in \Omega$, if $k \geq k_{0}$ and $k \varrho \leq t \leq(k+1) \varrho$, we have

$$
\frac{\log \left(1+V_{1}(t)\right)^{\theta}}{\log t} \leq \frac{\left.\left(1+\varepsilon_{u}\right) \log (k \varrho)\right)}{\log (k \varrho)}=1+\varepsilon_{u}
$$

Then

$$
\limsup _{t \rightarrow \infty} \frac{\log \left(1+V_{1}(t)\right)^{\theta}}{\log t} \leq 1+\varepsilon_{u} \text { a.s }
$$

Let $\varepsilon_{u} \rightarrow 0$, then

$$
\limsup _{t \rightarrow \infty} \frac{\log \left(1+V_{1}(t)\right)^{\theta}}{\log t} \leq 1 \text { a.s. }
$$


Hence

$$
\limsup _{t \rightarrow \infty} \frac{\log V_{2}(t)}{\log t} \leq \limsup _{t \rightarrow \infty} \frac{\log \left(1+V_{1}(t)\right)^{\theta}}{\log t} \leq \frac{1}{\theta} \text {, a.s }
$$

That is to say, for any small $0<\xi<1-\frac{1}{\theta}$, there exists a constant $T=T(\omega)$ and a set $\Omega_{\xi}$ as $P\left(\Omega_{\xi}\right) \geq 1-\xi$, and for $t \geq T, \omega \in \Omega_{\xi}$, we get

$$
\log V_{1}(t) \leq\left(\frac{1}{\theta}+\xi\right) \log t
$$

Therefore

$$
\limsup _{t \rightarrow \infty} \frac{V_{1}(t)}{t} \leq \limsup _{t \rightarrow \infty} \frac{t^{\frac{1}{\theta}+\xi}}{t}=0
$$

where together with the positivity of the solution, we get

$$
\lim _{t \rightarrow \infty} \frac{V_{1}(t)}{t}=0 \text { a.s }
$$

In which, we get (10). Finally, we obtain

$$
\begin{aligned}
\lim _{t \rightarrow \infty} \frac{S(t)}{t}=\lim _{t \rightarrow \infty} \frac{I(t)}{t}=\lim _{t \rightarrow \infty} \frac{Q(t)}{t}=\lim _{t \rightarrow \infty} \frac{e^{-\mu t} \int_{t-\tau_{1}}^{t} e^{\mu s} S(s) d s}{t}= \\
\lim _{t \rightarrow \infty} \frac{e^{-\mu t} \int_{t-\tau_{1}}^{t} e^{\mu s} I(s) d s}{t}, \\
\lim _{t \rightarrow \infty} \frac{e^{-\mu t \int_{t-\tau_{1}}^{t} e^{\mu s} Q(s) d s}}{t}=0 \text { a.s. Hence, this completes the proof. }
\end{aligned}
$$

Lemma 2 Let $(S(t), I(t), Q(t))$ be the solution of system (3) With any given initial condition $S\left(\xi_{1}\right) \geq 0, I\left(\xi_{2}\right) \geq 0$ and $Q\left(\xi_{3}\right) \geq 0$ for all $\xi_{1} \in\left[-\tau_{1}, 0\right), \xi_{2} \in\left[-\tau_{2}, 0\right)$ and $\xi_{3} \in\left[-\tau_{3}, 0\right)$ with $S(0), I(0)$ and $Q(0)>0$ then

$$
\lim _{t \rightarrow \infty} \frac{\int_{0}^{t} S(s) d B_{1}(s)}{t}=\lim _{t \rightarrow \infty} \frac{\int_{0}^{t} I(s) d B_{2}(s)}{t}=\lim _{t \rightarrow \infty} \frac{\int_{0}^{t} Q(s) d B_{3}(s)}{t}=0 .
$$

Proof Let $1<\theta<1+\frac{2 \mu}{\sigma}$ and denote $X_{i}(t)=\int_{0}^{t} x_{i}(s) d B_{i}(t)$ with $i=\{1,2,3\}$ and $x_{i}(t) \in\{S(t), I(t), Q(t)\}$.

According to the Burkholder-Davis-Gundy inequality (Theorem 7.3 [21]), we have

$$
\begin{aligned}
E\left[\sup _{0 \leq s \leq t}\left|X_{i}(s)\right|^{\theta}\right] & \leq C_{\theta} E\left[\int_{0}^{t} x_{i}^{2}(r) d r\right]^{\frac{\theta}{2}} \leq C_{\theta} t^{\frac{\theta}{2}} E\left[\sup _{0 \leq r \leq t} x_{i}^{2}(r)\right]^{\frac{\theta}{2}} \\
& \leq 2 M_{i} C_{\theta} t^{\frac{\theta}{2}}
\end{aligned}
$$


where $M_{i}$ are positive constants. Now, let $\varepsilon_{X_{i}}$ be an arbitrary positive constant, for $i=\{1 ; 2 ; 3\}$, Applying Doob's martingale inequality (Theorem 3.8 [21]) yields that

$$
P\left\{\omega: \sup _{k \leq t \leq(k+1)}\left|X_{i}(t)\right|>(k)^{\frac{1}{2}+\varepsilon_{X_{i}}+\frac{\theta}{2}}\right\} \leq \frac{E\left[\left|X_{i}(k+1)\right|^{\theta}\right]}{(k)^{\frac{1}{2}+\varepsilon_{X_{i}}+\frac{\theta}{2}}} \leq \frac{2 M_{i} C_{\theta}(k+1)^{\frac{\theta}{2}}}{(k)^{\frac{1}{2}+\varepsilon_{X_{i}}+\frac{\theta}{2}}}
$$

Thus, by Borel-Cantelli lemma, we get that for almost all $\omega \in \Omega$

$$
\sup _{k \leq t \leq(k+1)}\left|X_{i}(s)\right|^{\theta} \leq(k)^{\frac{1}{2}+\varepsilon_{X}+\frac{\theta}{2}}
$$

Verified for all finite $k$. Therefore, there exists a positive random integer $k_{0}(\omega)$, for almost all $\omega \in \Omega$, where (20) satisfied whenever $k \geq k_{0}$. Thus, for almost all $\omega \in \Omega$, if $k \geq k_{0}$ and $k \leq t \leq(k+1)$,

$$
\frac{\log \left|X_{i}(t)\right|^{\theta}}{\log t} \leq \frac{\left(\frac{1}{2}+\varepsilon_{X_{i}}+\frac{\theta}{2}\right) \log (k)}{\log (k)}=\frac{1}{2}+\varepsilon_{X_{i}}+\frac{\theta}{2}
$$

Then

$$
\limsup _{t \rightarrow \infty} \frac{\log \left|X_{i}(t)\right|}{\log t} \leq \frac{\frac{1}{2}+\varepsilon_{X_{i}}+\frac{\theta}{2}}{\theta}
$$

Letting $\varepsilon_{X_{i}} \rightarrow 0$, we get

$$
\lim _{t \rightarrow \infty} \frac{\log \left|X_{i}(t)\right|}{\log t} \leq \frac{1+\theta}{2 \theta}=\frac{1}{2}+\frac{1}{2 \theta}
$$

That is to say, for any small $0<\chi_{1}<\frac{1}{2}-\frac{1}{2 \theta}$. There exist a positive constant $T=T(\omega)$ and set $\Omega_{\chi_{1}}$ as $P\left(\Omega_{\chi_{1}}\right) \geq 1-\chi_{1}$ and for $t \geq T, \omega \in \Omega_{\chi_{1}}$, we get

$$
\ln \left|X_{i}\right| \leq\left(\frac{1}{2 \theta}+\chi_{1}\right) \ln t
$$

so

$$
\limsup _{t \rightarrow \infty} \frac{\left|X_{i}(t)\right|}{t} \leq \limsup _{t \rightarrow \infty} \frac{t^{\frac{1}{2 \theta}+\chi_{1}}}{t}=0
$$

Which together with $\lim \inf _{t \rightarrow \infty} \frac{\left|X_{i}(t)\right|}{t} \geq 0$, we get

$$
\lim _{t \rightarrow \infty} \frac{\left|X_{i}(t)\right|}{t}=0 \text { a.s. }
$$


yields that

$$
\lim _{t \rightarrow \infty} \frac{X_{i}(t)}{t}=0 \quad \text { a.s. }
$$

Therefore, we get (18). This finishes the proof.

\subsection{Extinction}

The main preoccupation in the study of dynamical behavior of epidemic models is how to control the disease dynamics in order to die out in long term. In this section, we shall establish sufficient conditions for extinction of the disease in the stochastic model (3). In the sequel, we set $\langle f(t)\rangle=\frac{1}{t} \int_{0}^{t} f(u) d u$, and we will investigate the behaviour of the stochastic epidemic model (3) according to a following stochastic threshold

$$
\mathcal{R}_{s}=\mathcal{R}_{0}-\frac{\sigma_{2}^{2}}{2\left(\mu+\alpha_{1}+\delta+\gamma\right)} .
$$

Theorem 2 Let $(S(t), I(t), Q(t))$ be a solution of system (3) with any initial value $S\left(\xi_{1}\right) \geq 0, I\left(\xi_{1}\right) \geq 0$ and $Q\left(\xi_{1}\right) \geq 0$ for all $\xi_{1} \in\left[-\tau_{1}, 0\right), \xi_{2} \in\left[-\tau_{2}, 0\right)$ and $\xi_{3} \in\left[-\tau_{3}, 0\right)$ with $S(0)>0, I(0)>0$ and $Q(0)>0$. If $\mathcal{R}_{s}<1$ then

$$
\limsup _{t \rightarrow \infty} \frac{\log (I(t))}{t} \leq\left(\mu+\alpha_{1}+\delta+\gamma\right)\left(\mathcal{R}_{s}-1\right)<0, \quad \text { a.s. }
$$

Moreover,

$$
\lim _{t \rightarrow \infty}\langle S(t)\rangle=\frac{A}{\mu+p\left(1-e^{-\mu \tau_{2}}\right)} \quad \text { a.s }
$$

and

$$
\lim _{t \rightarrow \infty}\langle Q(t)\rangle=0 \quad \text { a.s. }
$$

Proof We have

$$
\begin{aligned}
& d\left(S(t)+I(t)+\frac{\varepsilon e^{-\mu \tau_{3}}}{\mu+\alpha_{2}+\varepsilon} Q(t)+p e^{-\mu \tau_{1}} \int_{t-\tau_{1}}^{t} S(s) d s+\gamma e^{-\mu \tau_{2}} \int_{t-\tau_{2}}^{t} I(s) d s+\varepsilon e^{-\mu \tau_{3}} \int_{t-\tau_{3}}^{t} Q(s) d s\right) \\
& =\left[A-\left(\mu+p\left(1-e^{-\mu \tau_{1}}\right)\right) S(t)-\left(\mu+\alpha_{1}+\gamma\left(1-e^{-\mu \tau_{2}}\right)+\delta-\frac{\delta \varepsilon e^{-\mu \tau_{3}}}{\mu+\alpha_{2}+\varepsilon}\right) I(t)\right] d t+\sigma_{1} S d B_{1}(t) \\
& \quad+\sigma_{2} I d B_{2}(t)+\frac{\varepsilon e^{-\mu \tau_{3}}}{\mu+\alpha_{2}+\varepsilon} Q(t) d B_{3}(t) \\
& =\left[A-\left(\mu+p\left(1-e^{-\mu \tau_{1}}\right)\right) S(t)\right] d t+\sigma_{1} S(t) d B_{1}(t)+\sigma_{2} I(t) d B_{2}(t)+\frac{\sigma_{3} \varepsilon e^{-\mu \tau_{3}}}{\mu+\alpha_{2}+\varepsilon} Q(t) d B_{3}(t)
\end{aligned}
$$




$$
-\frac{\left(\mu+\alpha_{1}+\gamma\left(1-e^{-\mu \tau_{2}}\right)+\delta\right)\left(\mu+\alpha_{2}\right)+\varepsilon\left(\mu+\alpha_{1}+\gamma\left(1-e^{-\mu \tau_{2}}\right)+\delta\left(1-e^{-\mu \tau_{3}}\right)\right)}{\mu+\alpha_{2}+\varepsilon} I(t) d t .
$$

Then, we can get

$$
\begin{aligned}
& \frac{S(t)+I(t)+\frac{\varepsilon e^{-\mu \tau_{3}}}{\mu+\alpha_{2}+\varepsilon} Q(t)+p e^{-\mu \tau_{1}} \int_{t-\tau_{1}}^{t} S(s) d s+\gamma e^{-\mu \tau_{2}} \int_{t-\tau_{2}}^{t} I(s) d s+\varepsilon e^{-\mu \tau_{3}} \int_{t-\tau_{3}}^{t} Q(s) d s}{t} \\
& -\frac{S(0)+I(0)+\frac{\varepsilon e^{-\mu \tau_{3}}}{\mu+\alpha_{2}+\varepsilon} Q(0)+p e^{-\mu \tau_{1}} \int_{-\tau_{1}}^{0} S(s) d s+\gamma e^{-\mu \tau_{2}} \int_{-\tau_{2}}^{0} I(s) d s+\varepsilon e^{-\mu \tau_{3}} \int_{-\tau_{3}}^{0} Q(s) d s}{t} \\
& =A-\left(\mu+p\left(1-e^{-\mu \tau_{1}}\right)\right)\langle S(t)\rangle+\frac{\sigma_{1}}{t} S(t) d B_{1}(t)+\frac{\sigma_{2}}{t} I(t) d B_{2}(t)+\frac{\sigma_{3} \varepsilon e^{-\mu \tau_{3}}}{\left(\mu+\alpha_{2}+\varepsilon\right) t} Q(t) d B_{3}(t) \\
& -\left[\frac{\left(\mu+\alpha_{1}+\gamma\left(1-e^{-\mu \tau_{2}}\right)+\delta\right)\left(\mu+\alpha_{2}\right)+\varepsilon\left(\mu+\alpha_{1}+\gamma\left(1-e^{-\mu \tau_{2}}\right)+\delta\left(1-e^{-\mu \tau_{3}}\right)\right)}{\mu+\alpha_{2}+\varepsilon}\right]\langle I(t)\rangle .
\end{aligned}
$$

Thus

$$
\langle S(t)\rangle=\frac{A-\left[\frac{\left(\mu+\alpha_{1}+\gamma\left(1-e^{-\mu \tau_{2}}\right)+\delta\right)\left(\mu+\alpha_{2}\right)+\varepsilon\left(\mu+\alpha_{1}+\gamma\left(1-e^{-\mu \tau_{2}}\right)+\delta\left(1-e^{-\mu \tau_{3}}\right)\right)}{\mu+\alpha_{2}+\varepsilon}\right]\langle I(t)\rangle}{\left(\mu+p\left(1-e^{-\mu \tau_{1}}\right)\right)}-\phi(t),
$$

where

$$
\begin{aligned}
\phi(t)= & \frac{S(t)+I(t)+\frac{\varepsilon e^{-\mu \tau_{3}}}{\mu+\alpha_{2}+\varepsilon} Q(t)+p e^{-\mu \tau_{1}} \int_{t-\tau_{1}}^{t} S(s) d s+\gamma e^{-\mu \tau_{2}} \int_{t-\tau_{2}}^{t} I(s) d s+\varepsilon e^{-\mu \tau_{3}} \int_{t-\tau_{3}}^{t} Q(s) d s}{\left(\mu+p\left(1-e^{-\mu \tau_{1}}\right)\right) t} \\
& -\frac{S(0)+I(0)+\frac{\varepsilon e^{-\mu \tau_{3}}}{\mu+\alpha_{2}+\varepsilon} Q(0)+p e^{-\mu \tau_{1}} \int_{-\tau_{1}}^{0} S(s) d s+\gamma e^{-\mu \tau_{2}} \int_{-\tau_{2}}^{0} I(s) d s+\varepsilon e^{-\mu \tau_{3}} \int_{-\tau_{3}}^{0} Q(s) d s}{\left(\mu+p\left(1-e^{-\mu \tau_{1}}\right)\right) t} \\
& +\frac{1}{\left(\mu+p\left(1-e^{-\mu \tau_{1}}\right)\right)}\left(\frac{\sigma_{1}}{t} S(t) d B_{1}(t)+\frac{\sigma_{2}}{t} I(t) d B_{2}(t)+\frac{\sigma_{3} \varepsilon e^{-\mu \tau_{3}}}{\left(\mu+\alpha_{2}+\varepsilon\right) t} Q(t) d B_{3}(t)\right)
\end{aligned}
$$

From the strong law of large numbers and lemma 2,we have

$$
\lim _{t \rightarrow \infty} \phi(t)=0 \quad \text { a.s. }
$$

Applying Itô's formula, we get

$$
d \log (I(t))=\left[\beta S(t)-\left(\mu+\alpha_{1}+\delta+\gamma+\frac{\sigma_{2}^{2}}{2}\right)\right] d t+\sigma_{2} d B_{2}(t)
$$

From (26) and (29) we get

$$
\begin{aligned}
\frac{\log I(t)}{t} \leq & \frac{\beta A}{\mu+p\left(1-e^{-\mu \tau_{1}}\right)}-\left(\mu+\alpha_{1}+\delta+\gamma+\frac{\sigma_{2}^{2}}{2}\right) \\
& -\beta \phi(t)+\frac{\sigma_{2} B_{2}(t)}{t}+\frac{\log (I(0))}{t} .
\end{aligned}
$$


By the law of large number for martingales [21] and (28), it follows that for $\mathcal{R}_{s}<1$, we obtain

$$
\limsup _{t \rightarrow \infty} \frac{\log I(t)}{t} \leq\left(\mu+\alpha_{1}+\delta+\gamma\right)\left(\mathcal{R}_{s}-1\right)<0 \text { a.s }
$$

which leads to

$$
\lim _{t \rightarrow \infty} I(t)=0 \text { a.s. }
$$

From (26)

$$
\begin{aligned}
\lim _{t \rightarrow \infty}\langle S(t)\rangle= & \frac{A}{\mu+p\left(1-e^{\left.-\mu \tau_{1}\right)}\right.} \\
& -\frac{\left(\mu+\alpha_{1}+\gamma\left(1-e^{-\mu \tau_{2}}\right)+\delta\right)\left(\mu+\alpha_{2}\right)+\varepsilon\left(\mu+\alpha_{1}+\gamma\left(1-e^{-\mu \tau_{2}}\right)+\delta\left(1-e^{-\mu \tau_{3}}\right)\right)}{\left(\mu+\alpha_{2}+\varepsilon\right)\left(\mu+p\left(1-e^{\left.\left.-\mu \tau_{1}\right)\right)}\right.\right.} \lim _{t \rightarrow \infty}\langle I(t)\rangle \\
= & \frac{A}{\mu+p\left(1-e^{\left.-\mu \tau_{1}\right)}\right.} \text { a.s. }
\end{aligned}
$$

From the third equation of the system, it follows that

$$
\frac{Q(t)-Q(0)}{t}=\delta\langle I(t)\rangle-\left(\mu+\alpha_{2}+\varepsilon\right)\langle Q(t)\rangle+\frac{\sigma_{3}}{t} \int_{0}^{t} Q(s) d B_{3}(s) .
$$

Thus, it follows from Lemma 2 and (30) that

$$
\lim _{t \rightarrow \infty}\langle Q(t)\rangle=0 \text { a.s. }
$$

This finishes the proof.

\subsection{Persistence in mean}

In this section, to study the persistence of the disease, we will establish sufficient conditions to fulfill the conditions in the definition of persistence in mean in [14], we also need the following lemma presented in [14].

Lemma 3 Let $g \in C([0, \infty) \times \Omega,(0, \infty))$ and $G \in C([0, \infty) \times \Omega, \mathbb{R})$ such that $\lim _{t \rightarrow \infty} \frac{G(t)}{t}=0$ a.s. If for all $t \geq 0$

$$
\ln g(t) \geq \lambda_{0} t-\lambda \int_{0}^{t} g(s) d s+G(t) \text { a.s. }
$$

Then

$$
\liminf _{t \rightarrow \infty}\langle g(t)\rangle \geq \frac{\lambda_{0}}{\lambda} \text { a.s }
$$

where $\lambda_{0} \geq 0$ and $\lambda>0$ are two real numbers. 
Theorem 3 Let $(S(t), I(t), Q(t))$ be the solution of system (3) with any initial value $S\left(\xi_{1}\right) \geq 0, I\left(\xi_{1}\right) \geq 0$ and $Q\left(\xi_{1}\right) \geq 0$ for all $\xi_{1} \in\left[-\tau_{1}, 0\right), \xi_{2} \in\left[-\tau_{2}, 0\right)$ and $\xi_{3} \in\left[-\tau_{3}, 0\right)$ with $S(0)>0, I(0)>0$ and $Q(0)>0$. If $\mathcal{R}_{s}>1$ then

$$
\liminf _{t \rightarrow \infty}\langle I(t)\rangle=I^{*}>0
$$

where

$$
\begin{aligned}
& \qquad I^{*}=\frac{\left(\mu+\alpha_{2}+\varepsilon\right)\left(\mu+p\left(1-e^{-\mu \tau_{1}}\right)\right)\left(\mu+\alpha_{1}+\gamma+\delta\right)\left(\mathcal{R}_{s}-1\right)}{\beta\left[\left(\mu+\alpha_{1}+\gamma\left(1-e^{-\mu \tau_{2}}\right)+\delta\right)\left(\mu+\alpha_{2}\right)+\varepsilon\left(\mu+\alpha_{1}+\gamma\left(1-e^{-\mu \tau_{2}}\right)+\delta\left(1-e^{-\mu \tau_{3}}\right)\right)\right]}, \\
& \limsup _{t \rightarrow \infty}\langle S(t)\rangle=\frac{\mu+\alpha_{1}+\gamma+\delta+\frac{\sigma_{2}^{2}}{2}}{\beta} \\
& \text { and }
\end{aligned}
$$

$$
\liminf _{t \rightarrow \infty}\langle Q(t)\rangle=\frac{\delta I^{*}}{\left(\mu+\alpha_{2}+\varepsilon\right)}>0
$$

Proof It follows from (26) and (29) that

$$
\begin{aligned}
\log I(t)= & \left(\frac{\beta A}{\mu+p\left(1-e^{-\mu \tau_{1}}\right)}-\left(\mu+\alpha_{1}+\gamma+\delta+\frac{\sigma_{2}^{2}}{2}\right)\right) t \\
& -\frac{\beta\left[\left(\mu+\alpha_{1}+\gamma\left(1-e^{-\mu \tau_{2}}\right)+\delta\right)\left(\mu+\alpha_{2}\right)+\varepsilon\left(\mu+\alpha_{1}+\gamma\left(1-e^{-\mu \tau_{2}}\right)+\delta\left(1-e^{-\mu \tau_{3}}\right)\right)\right]}{\left(\mu+\alpha_{2}+\varepsilon\right)\left(\mu+p\left(1-e^{-\mu \tau_{1}}\right)\right)}\langle I(t)\rangle t \\
& +\sigma_{2} B_{2}(t)+\log I(0)-\beta t \phi(t) .
\end{aligned}
$$

Therefore, from (28) and lemma 3 , we get

$$
\liminf _{t \rightarrow \infty}\langle I(t)\rangle=I^{*}
$$

Thus

$$
\limsup _{t \rightarrow \infty}\langle S(t)\rangle=\frac{\mu+\alpha_{1}+\gamma+\delta+\frac{\sigma_{2}^{2}}{2}}{\beta} \text { a.s. }
$$

From the third equation of the system, we get

$$
\frac{Q(t)-Q(0)}{t}=\delta\langle I(t)\rangle-\left(\mu+\alpha_{2}+\varepsilon\right)\langle Q(t)\rangle+\frac{\sigma_{3}}{t} \int_{0}^{t} Q(s) d B_{3}(s) .
$$

By the vertue of lemma 2 and (31)

$$
\begin{aligned}
\liminf _{t \rightarrow \infty}\langle Q(t)\rangle & =\frac{\delta}{\mu+\alpha_{2}+\varepsilon} \liminf _{t \rightarrow \infty}\langle I(t)\rangle \\
& =\frac{\delta I^{*}}{\left(\mu+\alpha_{2}+\varepsilon\right)} \quad \text { a.s. }
\end{aligned}
$$


Therefore the conditions in the definition of persistence in mean [14] are verified. This completes the proof.

\section{The existence of stationary distribution}

The ergodicity is one of the most important proprieties which will result that the infectious disease will survive in a population, which means a relatively weak characteristic. In the following, we will give a definition of the stationary distribution of the stochastic delayed systems. Then, we will discuss the existence of stationary distribution and the ergodicity of the delayed stochastic system (3) by constructing a suitable Lyapunov functional and using the stochastic Lyapunov analysis methods.

We recall that for each $t \geq 0$ and probability measure $\mu$ on $\left(C\left([-\tau, 0] ; \mathbb{R}_{+}^{d}\right)\right.$, $\mathcal{M}[-\tau, 0])$, where $\mathcal{M}[-\tau, 0]$ is the associated Borel $\sigma$-algebra in $[-\tau, 0]$, consider the probability measure $\mu P_{t}$ on $\left(C\left([-\tau, 0] ; \mathbb{R}_{+}^{d}\right), \mathcal{M}[-\tau, 0]\right)$ defined by

$$
\left(\mu P_{t}\right)(\Delta)=\int_{C\left([-\tau, 0] ; \mathbb{R}_{+}^{d}\right)} P_{t}(x, \Delta) \mu(d x) \text {, for } \Delta \in \mathcal{M}[-\tau, 0]
$$

Definition 1 Stationary Distribution [15]

A stationary distribution for (4) is a probability measure $\pi$ on $\left(C\left([-\tau, 0] ; \mathbb{R}_{+}^{d}\right)\right.$, $\left.\mathcal{M}_{[-\tau, 0]}\right)$ such that $\left(\pi P_{t}\right)(\Delta)=\pi(\Delta)$ for all $t \geq 0$ and $\Delta \in \mathcal{M}_{[-\tau, 0]}$.

Theorem 4 Let $\mathcal{R}_{s}>1$, then for any initial conditions (5), stochastic delayed system (3) admits a stationary distribution $\pi$ (.), and the solution of system (3) is ergodic.

Proof The diffusion matrix of the stochastic delayed SIQR model (3)

$$
A(S, I, Q)=\left[\begin{array}{ccc}
\sigma_{1}^{2} S^{2} & 0 & 0 \\
0 & \sigma_{2}^{2} I^{2} & 0 \\
0 & 0 & \sigma_{3}^{2} Q^{2}
\end{array}\right]
$$

Let $\Gamma$ be any bounded domain in $\mathbb{R}_{+}^{3}$, then there exists a positive constant

$$
L_{0}=\min \left\{\sigma_{1}^{2} S^{2}, \sigma_{2}^{2} I^{2}, \sigma_{3}^{2} Q^{2},(S, I, Q) \in \bar{\Gamma}\right\}
$$

such that

$$
\begin{aligned}
\Sigma_{i, j=1}^{3} a_{i j}(S, I, Q) \xi_{i} \xi_{j} & =\sigma_{1}^{2} S^{2} \xi_{1}^{2}+\sigma_{2}^{2} I^{2} \xi_{2}^{2}+\sigma_{3}^{2} Q^{2} \xi_{3}^{2} \\
& \geq L_{0}|\xi|^{2},(S, I, Q) \in \bar{\Gamma}, \xi=\left(\xi_{1}, \xi_{2}, \xi_{3}\right) \in \mathbb{R}^{3}
\end{aligned}
$$

This leads to verify the first condition, where the smallest eigenvalue of the diffusion matrix $A(S, I, Q)$ is bounded away from zero. 
Next, we construct a $C^{2}-$ function $\tilde{V}\left(X_{t}, t\right)$ with $X_{t}=(S(t), I(t), Q(t))$ and a closed set $U_{\varepsilon} \in \mathbb{R}_{+}^{3}$ such that $\sup _{X \in \mathbb{R}_{+}^{3} \backslash U_{\varepsilon}} \mathcal{L} \tilde{V}<-\tilde{M}<0$, with $\tilde{M}$ is a positive constant and

$$
\mu-\frac{m \sigma^{2}}{2}>0
$$

where $m$ is a positive constant.

We define a Lyapunov functional as follows

$$
\hat{V}\left(X_{t}, t\right)=M \tilde{V}_{1}+\tilde{V}_{2}+\tilde{V}_{3}
$$

where

$$
\begin{aligned}
\tilde{V}_{1}= & -\left(\log I+\frac{\beta}{u+p\left(1-e^{-\mu \tau_{1}}\right)}\left(S+I+p e^{-\mu \tau_{1}} \int_{t-\tau_{1}}^{t} S(s) d s\right)\right), \\
\tilde{V}_{2}= & -\log S-\log Q, \\
\tilde{V}_{3}= & \frac{1}{m+1}\left(S+I+Q+p e^{-\mu t} \int_{t-\tau_{1}}^{t} e^{\mu s} S(s) d s\right. \\
& \left.+\gamma e^{-\mu t} \int_{t-\tau_{2}}^{t} e^{\mu s} I(s) d s+\varepsilon e^{-\mu t} \int_{t-\tau_{3}}^{t} e^{\mu s} Q(s) d s\right)^{m+1},
\end{aligned}
$$

and $M>0$ is a constant large enough verifying

$$
-M \tilde{\lambda}+D \leq-1
$$

with the terms $\tilde{\lambda}$ and $D$ defined later.

Moreover, $\tilde{V}\left(X_{t}, t\right)$ is a continuous function and have a minimum point $\left(S_{0}, I_{0}, Q_{0}\right)$ in the interior of $\mathbb{R}_{3}^{+}$. Therefore, Let $\tilde{V}: \mathbb{R}_{+}^{3} \rightarrow \mathbb{R}_{+}$be a nonnegative function such that

$$
\tilde{V}=\hat{V}(S, I, Q)-\hat{V}\left(S_{0}, I_{0}, Q_{0}\right)
$$

Applying Itô's formula on $\tilde{V}_{1}$, we obtain

$$
\begin{aligned}
\mathcal{L} \tilde{V}_{1} \leq & -\beta S+\left(\mu+\alpha_{1}+\gamma+\delta+\frac{\sigma_{2}^{2}}{2}\right) \\
& -\frac{A \beta}{\mu+p\left(1-e^{-\mu \tau_{1}}\right)}+\beta S+\frac{\beta\left(\mu+\alpha_{1}+\gamma+\delta\right)}{\mu+p\left(1-e^{-\mu \tau_{1}}\right)} I \\
:= & -\left(\mu+\alpha_{1}+\gamma+\delta\right)\left(\mathcal{R}_{s}-1\right)+\frac{\beta\left(\mu+\alpha_{1}+\gamma+\delta\right)}{\mu+p\left(1-e^{-\mu \tau_{1}}\right)} I \\
:= & -\tilde{\lambda}+\frac{\beta\left(\mu+\alpha_{1}+\gamma+\delta\right)}{\mu+p\left(1-e^{-\mu \tau_{1}}\right)} I,
\end{aligned}
$$


where $\tilde{\lambda}=\left(\mu+\alpha_{1}+\gamma+\delta\right)\left(\mathcal{R}_{s}-1\right)>0$, Using Itó's formula on $\tilde{V}_{2}$ and $\tilde{V}_{3}$, we get

$$
\begin{aligned}
\mathcal{L} \tilde{V}_{2}= & -\frac{A}{S}+\beta I+\left(\mu+p+\frac{\sigma_{1}^{2}}{2}\right)-\frac{p S\left(t-\tau_{1}\right) e^{-\mu \tau_{1}}}{S(t)} \\
& -\frac{\gamma I\left(t-\tau_{2}\right) e^{-\mu \tau_{2}}}{S(t)}-\frac{\varepsilon Q\left(t-\tau_{3}\right) e^{-\mu \tau_{1}}}{S(t)} \\
& -\frac{\varepsilon I(t)}{Q(t)}+\mu+\alpha_{2}+\varepsilon+\frac{\sigma_{3}^{2}}{2}, \\
\mathcal{L} \tilde{V}_{3}= & \tilde{V}_{3}^{m}\left(A-\mu(S+I+Q)-\alpha_{1} I-\alpha_{2} Q-\mu p e^{-\mu t} \int_{t-\tau_{1}}^{t} e^{\mu s} S(s) d s\right. \\
& \left.-\mu \gamma e^{-\mu t} \int_{t-\tau_{2}}^{t} e^{\mu s} I(s) d s-\mu \varepsilon e^{-\mu t} \int_{t-\tau_{3}}^{t} e^{\mu s} Q(s) d s\right) \\
& +\frac{m}{2} \tilde{V}_{3}^{m-1}\left(\sigma_{1}^{2} S^{2}+\sigma_{2}^{2} I^{2}+\sigma_{3}^{2} Q^{2}\right) \\
\leq & \tilde{V}_{3}^{m}\left(A-\mu \tilde{V}_{3}\right)+\frac{m \sigma^{2}}{2} \tilde{V}_{3}^{m+1} \\
\leq & B-\frac{1}{2}\left(\mu-\frac{m \sigma^{2}}{2}\right)\left(S^{m+1}+I^{m+1}+Q^{m+1}\right),
\end{aligned}
$$

where

$$
\begin{aligned}
B= & \sup _{(S, I, Q) \in \mathbb{R}_{+}^{3}}\left\{A \tilde{V}_{1}^{m}-\frac{1}{2}\left(\mu-\frac{m \sigma^{2}}{2}\right) \tilde{V}_{1}^{m+1}-\frac{1}{2}\left(\mu-\frac{m \sigma^{2}}{2}\right)\left(p e^{-\mu t} \int_{t-\tau_{1}}^{t} e^{-\mu s} S(s) d s\right.\right. \\
& \left.\left.+\gamma e^{-\mu t} \int_{t-\tau_{2}}^{t} e^{-\mu s} I(s) d s+\varepsilon e^{-\mu t} \int_{t-\tau_{3}}^{t} e^{-\mu s} Q(s) d s\right)\right\}
\end{aligned}
$$

Hence

$$
\begin{aligned}
\mathcal{L} \tilde{V} \leq & -M \tilde{\lambda}+\frac{M \beta\left(\mu+\alpha_{1}+\delta+\gamma\right)}{\mu+p\left(1-e^{-\mu \tau_{1}}\right)} I-\frac{A}{S}-\frac{\delta I(t)}{Q(t)} \\
& +B+\beta I+2 \mu+p+\alpha_{2}+\varepsilon+\frac{\sigma_{1}^{2}+\sigma_{3}^{2}}{2} \\
& -\frac{1}{2}\left[\mu-\frac{m \sigma^{2}}{2}\right]\left(S^{m+1}+I^{m+1}+Q^{m+1}\right) .
\end{aligned}
$$

Define a bounded set such that

$$
U_{\epsilon}=\left\{(S, I, Q) \in \mathbb{R}_{+}^{3}, \epsilon \leq S \leq \frac{1}{\epsilon}, \epsilon^{2} \leq I \leq \frac{1}{\epsilon}, \epsilon^{3} \leq Q \leq \frac{1}{\epsilon^{3}}\right\},
$$

where $0<\epsilon<1$ is a sufficiently small and such that

$$
-\frac{\min (A, \gamma)}{\epsilon}+D<-1
$$




$$
\begin{aligned}
& -\frac{1}{4}\left[\mu-\frac{m \sigma^{2}}{2}\right] \epsilon^{m+1}+E<-1, \\
& -\frac{1}{4}\left[\mu-\frac{m \sigma^{2}}{2}\right] \epsilon^{m+1}+F<-1, \\
& -\frac{1}{4}\left[\mu-\frac{m \sigma^{2}}{2}\right] \epsilon^{m+1}+G<-1,
\end{aligned}
$$

with $D, E, F$ and $G$ are positive constants, where the expressions are introduced after in the presented cases. Knowing that for a sufficiently small $\varepsilon$. We divide $\mathbb{R}_{+}^{3} \backslash U_{\varepsilon}$ into six domains, such that

$$
\begin{aligned}
& U_{1}=\left\{(S, I, Q) \in \mathbb{R}_{+}^{3}, 0<S<\epsilon\right\}, \quad U_{2}=\left\{(S, I, Q) \in \mathbb{R}_{+}^{3}, 0<I<\epsilon\right\}, \\
& U_{3}=\left\{(S, I, Q) \in \mathbb{R}_{+}^{3}, \varepsilon^{2}<I, 0<Q<\epsilon^{3}\right\}, \\
& U_{4}=\left\{(S, I, Q) \in \mathbb{R}_{+}^{3}, S>\frac{1}{\epsilon}\right\}, \quad U_{5}=\left\{(S, I, Q) \in \mathbb{R}_{+}^{3}, I>\frac{1}{\epsilon}\right\}, \\
& U_{6}=\left\{(S, I, Q) \in \mathbb{R}_{+}^{3}, Q>\frac{1}{\epsilon^{3}}\right\} .
\end{aligned}
$$

In the following, we should prove that $\mathcal{L} \tilde{V} \leq-1$ on $\mathbb{R}_{+}^{3} \backslash U_{\varepsilon}$, it means verifying it on the above six domains.

Case 1 If $(S, I, Q) \in U_{1}$, then

$$
\begin{aligned}
\mathcal{L} \tilde{V} \leq & -\frac{A}{S}+M \frac{\beta\left(\mu+\alpha_{1}+\alpha+\gamma\right)}{\mu+p\left(1-e^{-\mu \tau_{1}}\right)} I-\frac{1}{2}\left[\mu-\frac{m \sigma^{2}}{2}\right]\left(S^{m+1}+I^{m+1}+Q^{m+1}\right)+B \\
& +\beta I+2 \mu+p+\alpha_{2}+\varepsilon \frac{\sigma_{1}^{2}+\sigma_{3}^{2}}{2} \\
\leq & -\frac{A}{\varepsilon}+D
\end{aligned}
$$

where

$$
\begin{aligned}
D= & \sup _{(S, I, Q) \in \mathbb{R}_{+}^{3}}\left\{M \frac{\beta\left(\mu+\alpha_{1}+\delta+\gamma\right)}{\mu+p\left(1-e^{-\mu \tau_{1}}\right)} I-\frac{1}{2}\left[\mu-\frac{m \sigma^{2}}{2}\right]\right. \\
& \left.\left(S^{m+1}+I^{m+1}+Q^{m+1}\right)+\beta I+B+2 \mu+p+\alpha_{2}+\varepsilon+\frac{\sigma_{1}^{2}+\sigma_{2}^{3}}{2}\right\}
\end{aligned}
$$

According to (41), one can get that for a sufficiently small $\varepsilon$

$$
\mathcal{L} \tilde{V} \leq-1 \text { for any }(S, I, Q) \in U_{1}
$$


Case 2 If $(S, I, Q) \in U_{2}$, we get

$$
\begin{aligned}
\mathcal{L} \tilde{V} \leq & -M \lambda+M \frac{\beta\left(\mu+\alpha_{1}+\alpha+\gamma\right)}{\mu+p\left(1-e^{-\mu \tau_{1}}\right)} I-\frac{1}{2}\left[\mu-\frac{m \sigma^{2}}{2}\right]\left(S^{m+1}+I^{m+1}+Q^{m+1}\right) \\
& +B+\beta I+2 \mu+p+\alpha_{2}+\varepsilon+\frac{\sigma_{1}^{2}+\sigma_{3}^{2}}{2} \\
\leq & -M \tilde{\lambda}+D
\end{aligned}
$$

According to (37), one can get that for a sufficiently small $\varepsilon$

$$
\mathcal{L} \tilde{V} \leq-1 \text { for any }(S, I, Q) \in U_{2}
$$

Case 3 When $(S, I, Q) \in U_{3}$, it follows that

$$
\begin{aligned}
\mathcal{L} \tilde{V} \leq & -\frac{\delta I}{Q}+M \frac{\beta\left(\mu+\alpha_{1}+\delta+\gamma\right)}{\mu+p\left(1-e^{-\mu \tau_{1}}\right)} I-\frac{1}{2}\left[\mu-\frac{m \sigma^{2}}{2}\right]\left(S^{m+1}+I^{m+1}+Q^{m+1}\right) \\
& +\beta I+B+2 \mu+p+\alpha_{2}+\varepsilon+\frac{\sigma_{1}^{2}+\sigma_{3}^{2}}{2} \\
\leq & -\frac{\delta}{\epsilon}+D .
\end{aligned}
$$

By (41), we conclude that $\mathcal{L} \tilde{V} \leq-1$ on $U_{3}$

Case 4 When $(S, I, Q) \in U_{4}$, we get

$$
\begin{aligned}
\mathcal{L} \tilde{V} & \leq-\frac{1}{4}\left[\mu-\frac{m \sigma^{2}}{2}\right] S^{m+1}+E \\
& \leq-\frac{1}{4}\left[\mu-\frac{m \sigma^{2}}{2}\right] \epsilon^{m+1}+E
\end{aligned}
$$

where

$$
\begin{aligned}
E= & \sup _{(S, I, Q) \in \mathbb{R}_{+}^{3}}\left\{-\frac{1}{4}\left[\mu-\frac{m \sigma^{2}}{2}\right] S^{m+1}+\frac{M \beta\left(\mu+\alpha_{1}+\delta+\gamma\right)}{\mu+p\left(1-e^{-\mu \tau_{1}}\right)} I+\beta I\right. \\
& \left.+B+2 \mu+p+\alpha_{2}+\varepsilon-\frac{1}{2}\left[\mu-\frac{m \sigma^{2}}{2}\right]\left(I^{m+1}+Q^{m+1}\right)+\frac{\sigma_{1}^{2}+\sigma_{3}^{2}}{2}\right\} .
\end{aligned}
$$

By virtue of (42), we get that $\mathcal{L} \tilde{V} \leq-1$ for all $(S, I, Q) \in U_{4}$

Case 5 When $(S, I, Q) \in U_{5}$, we get

$$
\mathcal{L} \tilde{V} \leq-\frac{1}{4}\left[\mu-\frac{m \sigma^{2}}{2}\right] I^{m+1}+F
$$




$$
\leq-\frac{1}{4}\left[\mu-\frac{m \sigma^{2}}{2}\right] \epsilon^{m+1}+F,
$$

where

$$
\begin{aligned}
F= & \sup _{(S, I, Q) \in \mathbb{R}_{+}^{3}}\left\{-\frac{1}{4}\left[\mu-\frac{m \sigma^{2}}{2}\right] I^{m+1}+\frac{M \beta\left(\mu+\alpha_{1}+\delta+\gamma\right)}{\mu+p\left(1-e^{-\mu \tau_{1}}\right)} I+\beta I\right. \\
& \left.+B+2 \mu+p+\alpha_{2}+\varepsilon-\frac{1}{2}\left[\mu-\frac{m \sigma^{2}}{2}\right]\left(S^{m+1}+Q^{m+1}\right)+\frac{\sigma_{1}^{2}+\sigma_{3}^{2}}{2}\right\} .
\end{aligned}
$$

By virtue of (43), we get that $\mathcal{L} \tilde{V} \leq-1$ for all $(S, I, Q) \in U_{5}$

Case 6 When $(S, I, Q) \in U_{6}$, we get

$$
\begin{aligned}
\mathcal{L} \tilde{V} & \leq-\frac{1}{4}\left[\mu-\frac{m \sigma^{2}}{2}\right] Q^{m+1}+F \\
& \leq-\frac{1}{4}\left[\mu-\frac{m \sigma^{2}}{2}\right] \epsilon^{3 m+3}+F,
\end{aligned}
$$

where

$$
\begin{aligned}
F= & \sup _{(S, I, Q) \in \mathbb{R}_{+}^{3}}\left\{-\frac{1}{4}\left[\mu-\frac{m \sigma^{2}}{2}\right] Q^{m+1}+\frac{M \beta\left(\mu+\alpha_{1}+\delta+\gamma\right)}{\mu+p\left(1-e^{-\mu \tau_{1}}\right)} I+\beta I\right. \\
& \left.+B+2 \mu+p+\alpha_{2}+\varepsilon-\frac{1}{2}\left[\mu-\frac{m \sigma^{2}}{2}\right]\left(S^{m+1}+I^{m+1}\right)+\frac{\sigma_{1}^{2}+\sigma_{3}^{2}}{2}\right\} .
\end{aligned}
$$

It follows from (44), we get that $\mathcal{L} \tilde{V} \leq-1$ for all $(S, I, Q) \in U_{6}$. Hence, from (45), (48), (50), (51), (53) and (55), we get that for a sufficiently small $\epsilon$

$$
\mathcal{L} \tilde{V}(S, I, Q) \leq-1 \text { for all }(S, I, Q) \in \mathbb{R}_{+}^{3} \backslash U_{\epsilon}
$$

This means that if the solution $(S, I, Q) \in \mathbb{R}_{+}^{3} \backslash U_{\varepsilon}$ of the delayed stochastic epidemic model (3), the mean time $\tau_{x}$ at which a path issuing from $X_{t}$ reaches the set $U$ is finite, and $\sup _{X \in K} E^{x} \tau<\infty$ for every compact $K \subset \mathbb{R}_{+}^{3}$.

In addition, Theorem 1 shows that the stochastic epidemic model has a unique global positive solution and by the vertue of Theorem 3.9 in [21]. The solution of the stochastic delayed system (3) is bounded. Therefore, according to [15], these properties imply that Theorem 2.2.1 is verified. Hence, we can obtain that the delayed stochastic system (3) is ergodic and admits a unique stationary distribution. 


\section{Numerical simulations}

In order to simulate our theoretical results for Theorems 2 and 3, we illustrate the paths of the delayed deterministic epidemic model (1) and delayed stochastic epidemic model (3) using the Euler-Maruyama method to investigate numerically the results on the stochastic threshold $\mathcal{R}_{s}$.

Example 1 In this simulated example, choosing the initial value $(S(0), I(0), Q(0))=$ $(5,15,5)$, and the parameters values are $A=1, \mu=0.09, \beta=0.18, \gamma=$ $0.55, \delta=0.44, \varepsilon=0.6, p=0.2, \sigma_{1}=0.2, \sigma_{2}=0.85, \sigma_{3}=0.5, \alpha_{1}=0.4, \alpha_{2}=$ 0.02 , and $\tau_{1}=\tau_{2}=\tau_{3}=0.5$. We can calculate easily the basic reproduction rate $\mathcal{R}_{0}=1.2309$ and the stochastic threshold $\mathcal{R}_{s}=0.9868<1$. According to Theorem 2 the disease will go to extinction. In Fig. 1, the extinction of the disease is well observed in the illustration of the delayed stochastic system trajectories.

Example 2 In this simulated example, choosing the initial value $(S(0), I(0), Q(0))=$ $(10,0.1,0.1)$, we keep the same parameters as Example 1 and we change $\mu=$ $0.09, \beta=0.39, \gamma=0.55, \sigma_{2}=\sigma_{3}=0.4$. We alleviate the quarantine strategy by reducing the quarantined individuals rate to $\varepsilon=0.3$. We can calculate easily the basic reproduction rate $\mathcal{R}_{0}=2.9679$ and the stochastic threshold $\mathcal{R}_{s}=2.9077>1$. As observed in Fig. 2 the disease will persist in mean which support the conclusion of Theorem 3.

\section{Susceptibles}

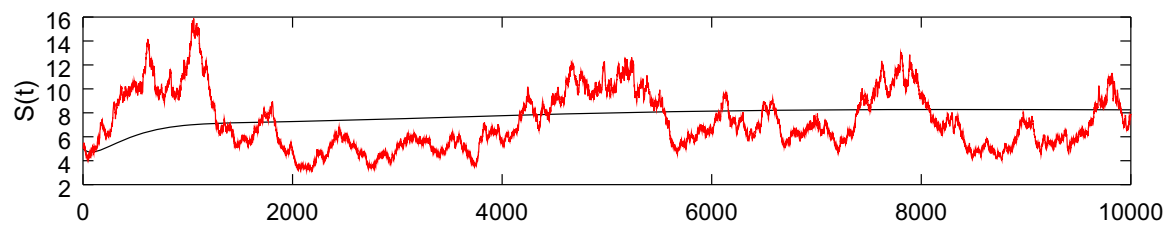

Infected

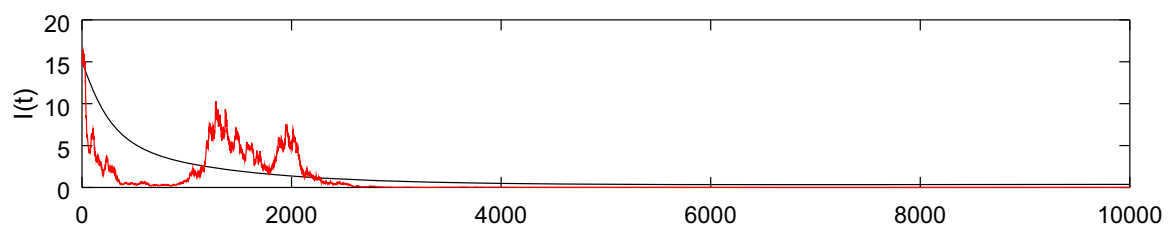

Quarantine

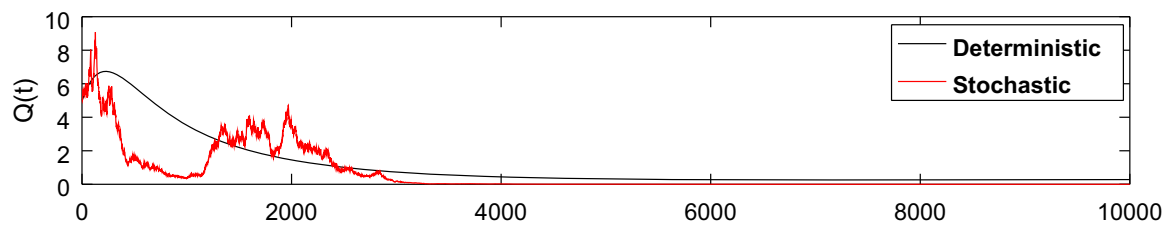

Fig. 1 Trajectories of stochastic and deterministic systems for example 1 

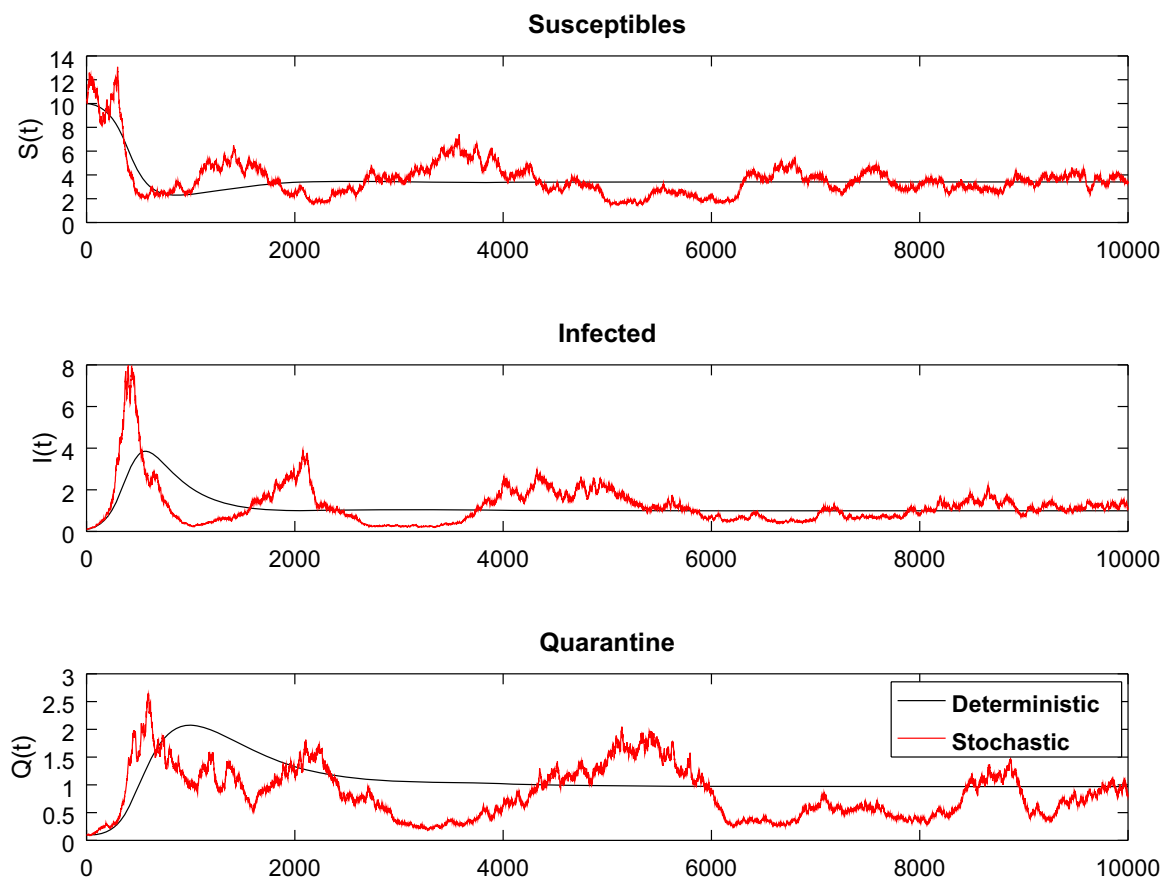

Fig. 2 Trajectories of stochastic and deterministic systems for Example 2

\section{Conclusion}

Since the quarantine has proven significant results in the control of the infectious diseases in a population such as the case of the recent pandemic COVID19 case. Therefore, we investigated in this work the behaviour of a delayed stochastic epidemic model with isolation, vaccination, elimination, temporary immunity.

In fact, we have studied the dynamics of a SIQR epidemic model including the notion of delay to describe the time efficiency of vaccine proposed in [9], the temporary loss of immunity [1] and the temporary loss of immunity after a quarantine is illustrated using the delay for quarantined individuals. We present a stochastic threshold $\mathcal{R}_{s}$ which is used to establish a sufficient condition for the extinction, persistence in mean and the existence of stationary distribution.

Acknowledgements The authors are very grateful to the Editor and the Reviewers for their helpful and constructive comments and suggestions. The authors are also thankful to the laboratory MAD (Management de l' agriculture Durable) of EST Sidi Bannour, the Faculty of sciences, Ibn Tofail University, Kenitra and Linnaeus University, V̈̈xjö for their help and support. 


\section{References}

1. Berrhazi, B., El Fatini, M., Laaribi, A.: A stochastic threshold for an epidemic model with BeddingtonDeAngelis incidence, delayed loss of immunity and Lévy noise perturbation. Phys. A 507, 312-320 (2018)

2. Berrhazi, B., El Fatini, M., Lahrouz, A., Settati, A., Taki, R.: A stochastic SIRS epidemic model with a general awareness-induced incidence. Phys. A 512, 968-980 (2018)

3. Cao, Z., Feng, W., Wen, X., Zu, L., Cheng, M.: Dynamics of a stochastic SIQR epidemic model with standard incidence. Phys. A 527, 121180, 12 (2019)

4. Cao, Z., Zhou, S.: Dynamical behaviors of a stochastic SIQR epidemic model with quarantine-adjusted incidence, p. 13. Discrete Dyn. Nat. Soc. (2018)

5. Caraballo, T., El Fatini, M., Sekkak, I., Taki, R., Laaribi, A.: A stochastic threshold for an epidemic model with isolation and a non linear incidence. Commun. Pure Appl. Anal. 19(5), 2513-2531 (2020)

6. Caraballo, T., Settati, A., El Fatini, M., Lahrouz, A., Imlahi, A.: Global stability and positive recurrence of a stochastic SIS model with Lévy noise perturbation. Phys. A 523, 677-690 (2019)

7. El Fatini, M., El Khalifi, M., Gerlach, R., Laaribi, A., Taki, R.: Stationary distribution and threshold dynamics of a stochastic SIRS model with a general incidence. Phys. A 534, 120696 (2019)

8. El Fatini, M., Sekkak, I.: Lévy noise impact on a stochastic delayed epidemic model with CrowlyMartin incidence and Crowding effect. Phys. A, Accepted (2019)

9. El Fatini, M., Sekkak, I., Laaribi, A.: A threshold of a delayed stochastic epidemic model with CrowlyMartin functional response and vaccination. Phys. A 520, 151-160 (2019)

10. Fan, K., Zhang, Y., Gao, S., Wei, X.: A class of stochastic delayed SIR epidemic models with generalized nonlinear incidence rate and temporary immunity. Phys. A 481, 198-208 (2017)

11. Guo, Y.: Stochastic regime switching SIS epidemic model with vaccination driven by lévy noise. Adv. Difference Equ. No. 375, 15 pp (2017)

12. Hattaf, K., Mahrouf, M., Adnani, J., Yousfi, N.: Qualitative analysis of a stochastic epidemic model with specific functional response and temporary immunity. Phys. A 490, 591-600 (2018)

13. Hethcote, H.W., Zhien, M., Shengbing, L.: Effects of quarantine in six endemic models for infectious diseases. Math. Biosci. 180, 141-160 (2002)

14. Ji, C., Jiang, D., O'Regan, D.: Threshold behaviour of a stochastic SIR model. Appl. Math. Comput. 38, 5067-5079 (2014)

15. Kinnally, M., Williams, R.: Stationary distributions for stochastic delay differential equations with non-negativity constraints. Electron. J. Probab. 15, 409-451 (2010)

16. Lan, G., Chen, Z., Wei, C., Zhang, S.: Stationary distribution of a stochastic SIQR epidemic model with saturated incidence and degenerate diffusion. Phys. A 511, 61-77 (2018)

17. Liu, Q., Chen, Q., Jiang, D.: The threshold of a stochastic delayed SIR epidemic model with temporary immunity. Phys. A 450, 115-125 (2016)

18. Liu, Q., Jiang, D., Hayat, T., Ahmad, B.: Analysis of a delayed vaccinated SIR epidemic model with temporary immunity and Lévy jumps. Nonlinear Anal. Hybrid Syst. 27, 29-43 (2018)

19. Liu, Q., Jiang, D., Shi, N., Hayat, T.: Dynamics of a stochastic delayed SIR epidemic model with vaccination and double diseases driven by Lévy jumps. Phys. A 492, 2010-2018 (2018)

20. Liu, Q., Jiang, D., Hayat, T., Alsaedi, A.: Dynamics of a stochastic multigroup SIQR epidemic model with standard incidence rates. J. Franklin Inst. 356(5), 2960-2993 (2019)

21. Mao, X.: Stochastic differential equations and applications. Second edn. Horwood Publishing Limited, Chichester, 2008, pp. xviii+422, ISBN 978-1-904275-34-3 (2010)

22. Qun, L., Daqing, J., Ningzhong, S.: Threshold behavior in a stochastic SIQR epidemic model with standard incidence and regime switching. Appl. Math. Comput. 316, 310-325 (2018)

23. Ruschel, S., Tiago, P., Serhiy, Y., Lai-Sang, Y.: An SIQ delay differential equations model for disease control via isolation. J. Math. Biol. 79(1), 249-279 (2019)

24. Wang, L.-L., Huang, N.-J., O'Regan, D.: Dynamics of a stochastic SIQR epidemic model with saturated incidence rate. Filomat 32(15), 5239-5253 (2018)

25. Zhang, X.-B., Huo, H.-F., Xiang, H., Li, D.: The dynamical behavior of deterministic and stochastic delayed SIQS model. J. Appl. Anal. Comput. 8(4), 1061-1084 (2018)

26. Zhang, X.-B., Huo, H.-F., Xiang, H., Meng, X.-Y.: Dynamics of the deterministic and stochastic SIQS epidemic model with non linear incidence. Appl. Math. Comput. 243, 546-558 (2014)

27. Zhang, X.-B., Huo, H.-F., Xiang, H., Shi, Q., Li, D.: The threshold of a stochastic SIQS epidemic model. Phys. A 482, 362-374 (2017) 
28. Zhang, X., Yuan, R.: The existence of stationary distribution of a stochastic delayed chemostat model. Appl. Math. Lett. 93, 15-21 (2019)

29. Zhang, Q., Zhou, K.: Stationary distribution and extinction of a stochastic SIQR model with saturated incidence rate. Math. Probl. Eng., Art. ID 3575410, 12 pp (2019)

30. Zhu, Z., Leung, H.: Optimal synchronization of chaotic systems in noise. IEEE Trans. Circ. Syst. I Fund. Theory Appl. 46, 1320-1329 (1999)

Publisher's Note Springer Nature remains neutral with regard to jurisdictional claims in published maps and institutional affiliations. 